\title{
Hysteresis Modeling of Robotic Catheters based on Long Short-Term Memory Network for Improved Environment Reconstruction
}

\author{
Di Wu ${ }^{1,2, *}$, Yao Zhang ${ }^{1, *}$, Mouloud Ourak ${ }^{1}$, Kenan Niu ${ }^{1}$, Jenny Dankelman ${ }^{2}$, \\ and Emmanuel Vander Poorten ${ }^{1}$
}

\begin{abstract}
Catheters are increasingly being used to tackle problems in the cardiovascular system. However, positioning precision of the catheter tip is negatively affected by hysteresis. To ensure tissue damage due to imprecise positioning is avoided, hysteresis is to be understood and compensated for. This work investigates the feasibility to model hysteresis with a Long ShortTerm Memory (LSTM) network. A bench-top setup containing a catheter distal segment was developed for model evaluation. The LSTM was first tested using four groups of test datasets containing diverse patterns. To compare with the LSTM, a Deadband Rate-Dependent Prandtl-Ishlinskii (DRDPI) model and a Support Vector Regression (SVR) model were established. The results demonstrated that the LSTM is capable of predicting the tip bending angle with sub-degree precision. The LSTM outperformed the DRDPI model and the SVR model by $60.1 \%$ and $36.0 \%$, respectively, in arbitrarily varying signals. Next, the LSTM was further validated in a 3D reconstruction experiment using Forward-Looking Optical Coherence Tomography (FLOCT). The results revealed that the LSTM was able to accurately reconstruct the environment with a reconstruction error below $0.25 \mathrm{~mm}$. Overall, the proposed LSTM enabled precise free-space control of a robotic catheter in the presence of severe hysteresis. The LSTM predicted the catheter tip response precisely based on proximal input pressure, minimizing the need to install sensors at the catheter tip for localization.
\end{abstract}

Index Terms-coronary artery disease, robotic catheter, hysteresis, modeling, LSTM, pneumatic artificial muscle.

\section{INTRODUCTION}

C ORONARY artery disease (CAD) is one of the most prevalent forms of heart disease. One-third of deaths over 35 years old were caused by CAD [1]. CAD reduces blood flow to the heart muscle because of plaque buildup in the coronary arteries. If left untreated, CAD may cause chest pain, unusual fatigue, and even sudden death [2]. A common procedure for treating CAD patients is Percutaneous Coronary Intervention (PCI), which is less invasive than surgeries. During PCI procedure, catheters are steered to recanalize

* These authors contributed equally to this work.

Manuscript received: October, 14, 2020; Revised: January, 15, 2021; Accepted: February, 3, 2021.

This paper was recommended for publication by Editor Pietro Valdastri upon evaluation of the Associate Editor and Reviewers' comments. This work was supported by European Union's Horizon 2020 research and innovation programme under the Marie Sklodowska-Curie grant agreement No. 813782

${ }^{1}$ D. Wu, Y. Zhang, M. Ourak, K. Niu, and E. Vander Poorten are with the Department of Mechanical Engineering, KU Leuven, 3001 Leuven, Belgium email: \{di.wu, yao.zhang, kenan.niu, mouloud.ourak, emmanuel.vanderpoorten\}@kuleuven.be

${ }^{2} \mathrm{D}$. Wu and J. Dankelman are with the Faculty of Mechanical, Maritime and Materials Engineering, Delft University of Technology, 2628 CD Delft, the Netherlands email: \{di.wu, j.dankelman\}@tudelft.nl

Digital Object Identifier (DOI): see top of this page. the occlusion. Due to the tortuosity of the aorta, the fragile and deformable nature of the vessels as well as heartbeat, good maneuverability and controllability of the catheters are imperative. Robotic catheters, which can achieve accurate and high-dexterity control compared to conventional manual catheters, emerged to address these demands [3].

Robotic catheters can be operated based on various working principles [4]. Cable-driven technology is one of the most popular driving principles for robotic catheters. The cables, which are routed over the entire length of the catheter, undergo quite some friction with their guiding tubes. Consequently, reaching a high bandwidth is generally difficult. This hinders the deployment of the cable-driven technology in scenarios in which good responsiveness is desirable. From the 1950s onwards, Pneumatic Artificial Muscle (PAM) became popular for many applications due to its high operation bandwidth, easy fabrication, and low-cost [5]. PAMs also show good promise for being used in interventional instruments, in which precise maneuvers are required [6]. Therefore, this paper focuses on catheter actuation with embedded PAMs.

Whether actuated by cables or by PAMs, precise control of catheters or, in a broad sense, flexible surgical robots is challenging. With hysteresis being a primary source of imprecision, hysteresis establishes a complex non-linear multi-valued relation between input commands and the response of the catheter distal tip. This multi-valued relation complicates navigation and results in inaccurate manipulation and positioning of the catheter tip. Inadequate positioning of the relatively acute tip could induce vessel wall damage or thrombus creation. To solve this issue, several methods have been explored in the past (Table I). The use of external sensors as feedback to implement a closed-loop control was presented in [7], [12]. However, mounting sensors at the distal end is challenging due to spatial restrictions and sterilization requirements [8]. Some previous research also demonstrated the feasibility to use imaging techniques as feedback to minimize hysteresis [9], [10]. Baek et al. further integrated these imaging-based methods with kinematic models [11]. Amongst others, analytic models for identifying hysteresis is one of the most popular research fields ([17] - [19]). An open-loop controller could be designed based on the inversion of the identified models. Nevertheless, analytic models require a large number of parameters which would lead to a cumbersome identification process. It's worth noting that, except for [19], all the above-mentioned research tackled hysteresis in cable-driven systems, while the study on hysteresis identification in fluidics-driven systems was scarce. Deep learning techniques have recently gained interest as 
Table I

METHODS TO MINIMIZE HYSTERESIS IN FLEXIBLE SURGICAL ROBOTS FROM PREVIOUS LITERATURE

\begin{tabular}{cccc}
\hline \hline Authors and publication year & Device & Actuation/Transmission Principle & Methods \\
\hline Bardou et al., 2012 [7] & Endoscope & Cable-Driven (CD) & Feedback from Electromagnetic (EM) sensor \\
Cabras et al., $2017[9]$ & Endoscope & CD & Locating catheter tip using imaging modalities \\
Reilink t al., 2013 [10] & Endoscope & CD & Locating catheter tip using imaging modalities \\
Baek et al., 2020 [11] & Multi-arm Flexible Surgical Robot & Tendon-sheath mechanism (TSM) & Computer vision + kinematic model \\
Do et al., 2014 [17] & Endoscope & TSM & Bouc-Wen model \\
Omisore et al., 2018 [18] & Cardiovascular Catheter & CD & Adaptive Backlash Model \\
Legrand et al., 2020 [19] & Fetoscope & Mckibben muscle & Generalized Prandtl-Ishlinskii Model \\
Xu et al., 2017 [20] & Serpentine Manipulator & TSM & Machine learning (regression) \\
Porto et al., 2019 [8] & Endoscope & CD & Machine learning + Inverse kinematic Model \\
\hline
\end{tabular}

they allow to avoid intricate identification processes by training an artificial neural network. Up to now, few works have attempted to use machine or deep learning for hysteresis modeling. Xu et al. [20] employed regression methods to learn the inverse kinematics model of a serpentine surgical manipulator. Porto et al. [8] used machine learning to produce position control of a flexible surgical robot. Both works dealt with cable-driven robots, and adopted traditional machine learning methods but did not take benefit of recent advances in deep learning. Several researchers used various deep learning approaches to model force hysteresis targeting generic [13], [16] and medical applications [14], [15]. In this work we tackle hysteresis in positioning tasks, whereas force tasks - as described in [13]-[16] form the topic of furher investigations and is out of the scope of this work. In this paper, we propose to use a Long Short-Term Memory (LSTM) network to model the hysteresis in a catheter system. It is shown that the proposed method: 1) is able to predict the catheter distal response merely based on proximal input commands without including sensory feedback; 2) has a high modeling accuracy compared to other existing analytic models and machine learning models; 3) does not require a large amount of data and has a fast training procedure. The LSTM allows catheters to be steered precisely during endovascular interventions. A further contribution is that we use the developed model together with OCT-based distance sensing to reconstruct a 3D artificial environment. The reconstruction error is analyzed and discussed in detail and directions for further work are provided. This paper is structured as follows: Section II describes the structure of the LSTM as well as an experimental setup that was used for data collection and algorithm validation. Section III introduces the designed experiments aimed for validating the performance of the LSTM. The experimental results and related discussions are presented as well in this section. Section IV concludes the work and proposes some future work on catheter control.

\section{Methods}

\section{A. Long Short-Term Memory Network}

A system is said to exhibit hysteresis if it has a sort of memory. This means that the output at a certain moment is not only determined by the corresponding input but also by the past inputs [22].

It happens that an LSTM was proposed as a tool to process sequential information and take historical information into account [23]. It is therefore not so illogical to consider the use of LSTM to model hysteretic behavior.

$$
\begin{gathered}
f^{(t)}=\sigma\left(W_{f} p^{(t)}+U_{f} h^{(t-1)}+b_{f}\right) \\
i^{(t)}=\sigma\left(W_{i} p^{(t)}+U_{i} h^{(t-1)}+b_{i}\right) \\
\widetilde{c}^{(t)}=\tanh \left(W_{g} p^{(t)}+U_{g} h^{(t-1)}+b_{g}\right) \\
c^{(t)}=f^{(t)} * c^{(t-1)}+i^{(t)} * \widetilde{c}^{(t)} \\
o^{(t)}=\sigma\left(W_{o} p^{(t)}+U_{o} h^{(t-1)}+b_{o}\right) \\
h^{(t)}=\tanh \left(c^{(t)}\right) * o^{(t)}
\end{gathered}
$$

LSTM can be viewed as a stack of LSTM cells that contain a number of units in an LSTM cell (see Fig. 1). Each LSTM cell is equipped with an input gate, an output gate, and a forget gate that control the information flow. The information in a unit is processed following (1) [23]. In (1), $p^{(t)}$ is the input pressure to a Pneumatic Artificial Muscle (PAM) at time t. The variable $\sigma$ is the sigmoid function with an output range of $[0,1]$. It regulates the percentage of information flows through each gate. Another activation function is the tanh, whose outputs range from $[-1,1]$. The forget gate $f$ determines which memory in the previous cell state $c^{(t-1)}$ is retained. The input gate $i$ and the input modulation gate $\widetilde{c}$ are combined to update the cell state. A new cell state $c^{(t)}$ is created afterwards. The cell state $c$ stores selective information that the LSTM processes all along, thus a long-term memory in the LSTM can be established. The output gate $o$ works as a filter. It regulates which part of information from the new cell state $c^{(t)}$ is output and transferred to the hidden state $h^{(t)}$. The matrix $W, U$ and $b$, with different subscripts, represent the weights and biases in each gate. These gates keep track of the dependencies between the elements in the input sequence.

\section{B. Experimental Setup}

In order to collect data for training and testing the LSTM, a bench-top experimental setup was built. A schematic diagram of this setup is illustrated in Fig. 2. This setup contains a one degree-of-freedom (DOF) unidirectional catheter distal segment with an embedded PAM (Fig. 2). The catheter is fabricated out of Nitinol using metal laser cutting technology and has a diameter of $4.4 \mathrm{~mm}$. The catheter diameter will be minimized in the future for the PCI. The custom-made artificial muscle, which is made up of a bladder, an uninflatable tube, braids and ferrules, is used to actuate the catheter. This artificial muscle has an initial diameter of $2.1 \mathrm{~mm}$ and a length of 129 $\mathrm{mm}$ in undeflected condition. By increasing the pressure, the muscle contracts and applies, through a steer cable that is attached off-centered at the catheter tip, a bending moment on the catheter tip. In order to accurately control the input pressure, the pressure is fed by an air supply, via a proportional pressure valve (Festo, Germany) to the artificial muscle in cascade (Fig. 2). The proportional valve receives control 


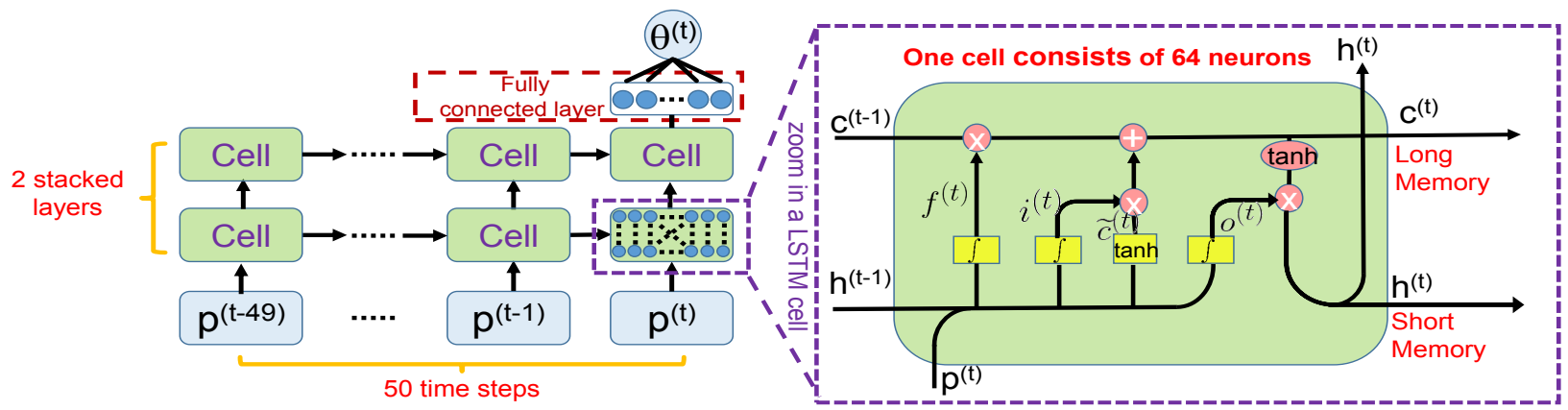

Figure 1. Left: a stacked 2-layer LSTM that consists of multiple LSTM cells, is unrolled into multiple time steps. In this work, LSTM cell is comprised of 64 neurons. A fully connected layer is added after the LSTM cell at time $t$. Right: each LSTM unit has three gates, i.e., input gate $i$, output gate $o$, and forget gate $f$ that regulate information flow according to (1). A window size of 50 is used to incorporate the input pressure $\mathrm{p}$ that was applied in the last 50 time steps. All this information is used to predict the future bending angle $\theta$.

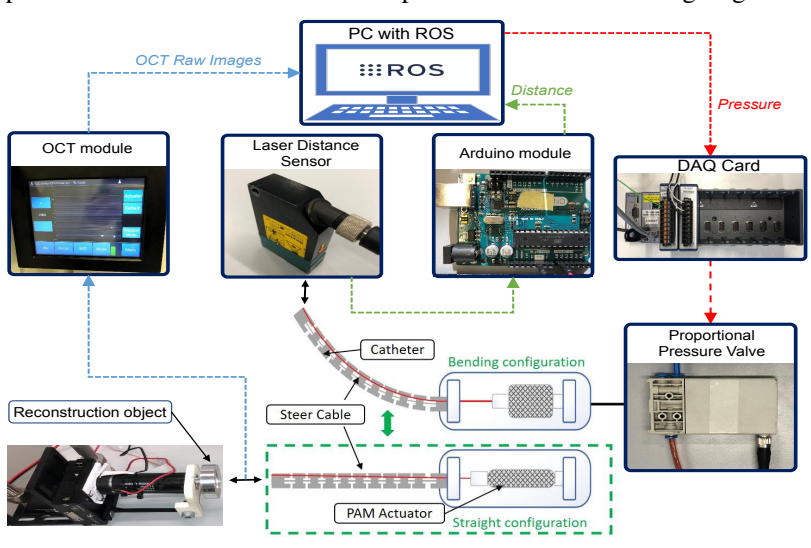

Figure 2. A PAM-driven catheter segment is controlled by a proportional pneumatic valve, which receives a command signal from a PC through a NI ${ }^{\circledR}$ CompactRIO system. The resulting catheter tip bending angle is captured by a laser sensor. A ROS-based GUI is created for pressure control and data collection.

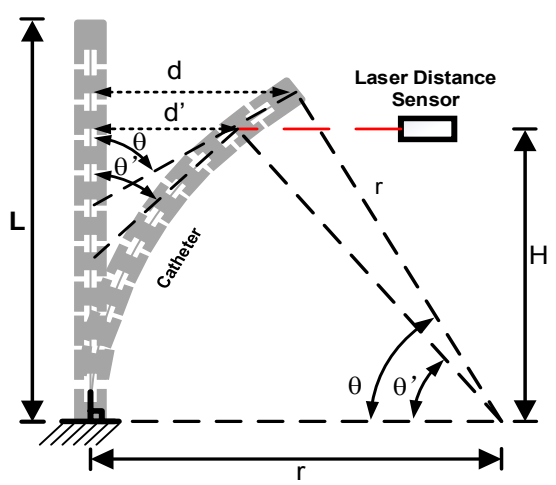

Figure 3. The catheter tip displacement $d^{\prime}$ captured by the laser distance sensor is converted to bending angle $\theta$ using (2) - (4) based on a constant curvature model [21] assumption.

signals from an analog output module NI-9263 (NI, Texas, USA). A pressure sensor (21Yseries, Keller, Switzerland) is installed in series with the above-mentioned circuit in order to measure the pressure value. A laser photoelectric sensor (OADM 12I6460/S35A, Baumer Group, Switzerland) is used to measure the distance $s$ (unit: $\mathrm{mm}$ ) from the sensor to the catheter tip with a sampling frequency of $250 \mathrm{~Hz}$. The measured voltage output $U$ (unit: V) from the laser sensor can be converted to the catheter tip bending angle $\theta$ (unit: deg) using following relation:

$$
\begin{gathered}
s_{i}=m U_{i}+n \\
d_{i}^{\prime}=\max \left(s_{1}, s_{2}, \cdots, s_{N}\right)-s_{i}
\end{gathered}
$$

$$
\theta_{i}=\frac{2 \cdot d_{i}^{\prime} \cdot L}{H^{2}+d_{i}^{\prime 2}} \cdot \frac{180}{\pi}
$$

In (2) - (4), subscript $i$ indicates the $i$-th sample in a group and the $N$ represents the number of samples in a data group. The voltage $U$ that is measured by the laser photoelectric sensor is proportional to the distance $s$ to the measured object, thus the $m=4.05(\mathrm{~mm} / \mathrm{V})$ and $n=29.36(\mathrm{~mm})$ in $(2)$ was identified only once prior to data collection. The maximum distance between the sensor and the catheter tip is reached when the catheter is straight, while the minimum distance is reached when the maximum pressure is given to the catheter. Referencing the straight configuration as zero displacement, then the displacement of catheter tip $d^{\prime}$ can be calculated by subtracting the measured distance $s$ from the maximum distance $\max \left(s_{1}, s_{2}, \cdots, s_{N}\right)$ between the catheter tip and the laser sensor as expressed in (3). Afterwards, as shown in Fig. 3, the catheter bending angle $\theta$ can be obtained based on a constant curvature model [21] assumption, in which the gravitational and inertial forces of the catheter segment could be assumed to be negligible. The data from the distance sensor is collected by an Arduino microcontroller, and then sent back to the PC via a Robot Operating System (ROS) topic. A Graphical User Interface (GUI) is created in ROS to facilitate the users to switch among different excitation signals which are detailed in Section II-C.

\section{Training Data Acquisition}

In order to provide the LSTM with data containing diverse excitation patterns, descending sinusoidal pressure with zero baseline described in (5) and with non-zero baseline described in (6) were sent to the setup to generate multi-loop hysteresis (Fig. 4).

$$
\begin{array}{ll}
p_{1}(t)=A e^{-\tau t}\left(\sin \left(2 \pi f t-\frac{\pi}{2}\right)+1\right) & {[\mathrm{bar}]} \\
p_{2}(t)=A e^{-\tau t}\left(\sin \left(2 \pi f t-\frac{\pi}{2}\right)\right)+A & {[\mathrm{bar}]}
\end{array}
$$

The amplitude $A$ of both signals is set to 1.5 to achieve a maximum amplitude of 3 bars. In (5) and (6), the variable $f$ is the excitation frequency in $\mathrm{Hz}$. Input frequencies up to $1 \mathrm{~Hz}$ are investigated in this work. Hysteresis behavior depends on both the input frequency and the amplitude of the excitation signal [25], different excitation frequencies are included in the training data, namely the frequencies of the excitation signal are set to $0.2,0.4,0.6$ and 0.8 in the training data set. The 

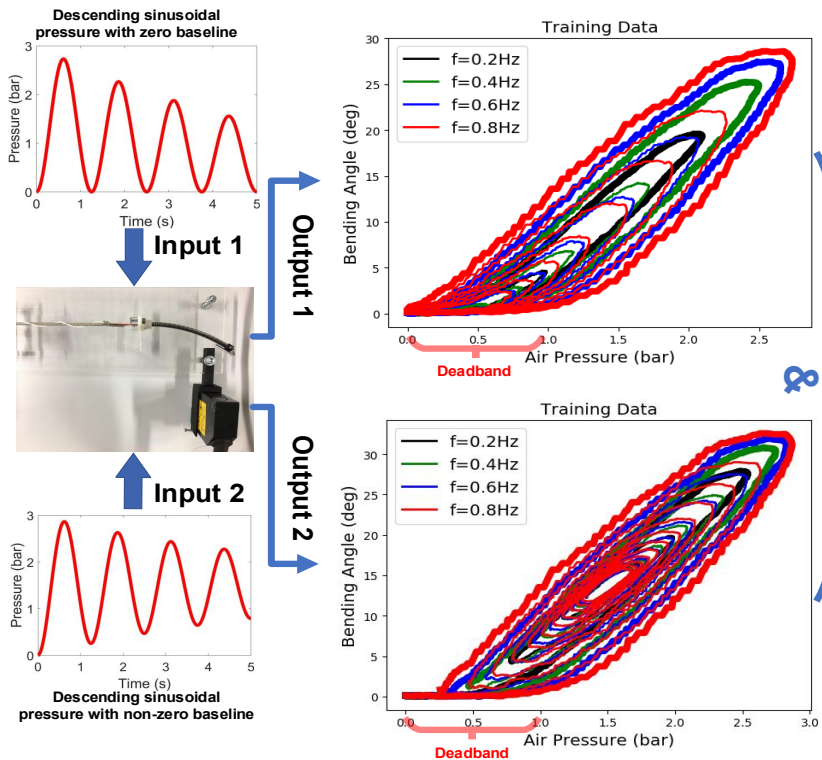

Figure 4. The training data are collected from the experimental setup: bending angle (deg) are visualized as a function of the input pressures (bar): (Output1): 4 groups of data with zero baseline are collected based on combinations of $f=0.2,0.4,0.6,0.8$ and time constant $\tau=0.15$ following (5); (Output 2 ): 4 groups of data with non-zero baseline are collected following (6) based on the same parameters as in output 1 . Thick lines represent the major hysteresis loop with maximum excitation, while thinner ones indicate the minor loops.

time constant $\tau$ was chosen with only one value $\tau=0.15$ to generate multi-loop hysteresis.

Two types of training data (bending angle - pressure) featuring major and minor loops were obtained as shown in Fig. 4. In total 26798 samples were acquired in the training data set. The training data reveals widening hysteresis loops with raising excitation frequencies. One can also observe deadbands at the bottom of the loops (Fig. 4). It is noteworthy that the hysteresis does not only come from the PAM, but also due to friction from e.g. relative movements of the steer cable and NiTi tube during bending, the compressibility of air, the compliance of the pneumatic tubes, the nonlinear behaviour of the employed material (Nitinol) and of the valves that were used. All these phenomena contribute to the resulting asymmetric, saturated, rate-dependent hysteresis behavior.

\section{Hyperparameters of the LSTM and Network Training}

A 2-layer stacked LSTM (see Fig. 1) was adopted. According to a previous pilot study [24], a window size equal to 50 was shown a good performance while maintaining a low computational cost. Therefore, the input pressure were segmented into a window size of 50. In other words, the sequential pressure data $p^{(t-49)}, p^{(t-48)}, \ldots p^{(t)}$ were entered into the LSTM to predict one bending angle $\theta^{(t)}$. Zeros were padded on the left of the input sequence for predicting the first 49 bending angles. A fully connected layer (input dimensions $=64$, output dimension $=1$ ) was added after the last LSTM cell. To increase the learning efficacy, all the training data were normalized between $[-1,1]$ to match the range of the activation function in (1). The hyperparameters of the LSTM are listed in Table II. The LSTM is simple and fast with only 2 hidden layers and 64 neurons per layer. These two hyperparameters were chosen and tested empirically, as adding more layers or neurons did not only led to worse fitting, but also increased the computational cost and the needed training time. The total number of trainable parameters is 50497. The network was implemented in Pytorch, an open source machine learning framework. The training was performed using an 4 GB NVIDIA CUDA-capable GPU. The LSTM was trained for 50 epochs and the whole training time was around 10 minutes.

\section{E. Modeling Evaluation Methods}

Three metrics i.e. the Maximum Absolute Error (MAE), the Root Mean Square Error (RMSE) and the Normalized Root Mean Square Error (NRMSE) are used to quantitatively evaluate the model performance in this paper. The MAE that is computed following (7) measures the maximum absolute difference between the predicted bending angles and ground truth among all the samples:

$$
M A E=\max \left\{\left|\hat{\theta}_{i}-\theta_{i}\right|\right\}, i=1,2, \ldots, N
$$

$\mathrm{N}$ is the number of sample points in each group of test data. The RMSE following (8) calculates the square root of the square difference between the predicted bending angles and the ground truth.

$$
R M S E=\sqrt{\frac{\sum_{i=1}^{N}\left(\hat{\theta}_{i}-\theta_{i}\right)^{2}}{N}}, i=1,2, \ldots, N
$$

The NRMSE relates the RMSE to the observed range of the variables, and it is defined as:

$$
N R M S E=\frac{R M S E}{\theta_{\max }-\theta_{\min }}
$$

\section{EXPERIMENTS AND RESULTS}

\section{A. Preliminary Evaluation of the LSTM}

In the preliminary evaluation, a well-trained LSTM was tested on four types of signals (see Fig. 5). The LSTM, similar to other neural networks, consists of a huge amount of weights and biases that are distributed in each layer. The output of the LSTM is derived by multiplying the inputs with the embedded weights and biases. Therefore, the experiments in this section was merely carried out once since a LSTM would predict the same results if the input remains the same.

A state-of-the-art analytic model called Deadband RateDependent Prandtl-Ishlinskii (DRDPI) model proposed in [25] was established for comparison to the LSTM. The DRDPI model is a sophisticated and practical model that takes into account the impact of frequency on the pattern of the hysteresis. In addition, deadband operators in this model allow it to model asymmetric hysteresis as well as saturation-exhibiting behaviour that appears at the bottom of the hysteresis loops (see Fig. 4). Therefore, the DRDPI model is fully adapted to model the hysteresis originating from the entire PAM-driven catheter system. The parameters of the DRDPI model were identified using a genetic optimization algorithm in MATLAB ${ }^{\circledR}$ Toolbox. The training data remained the same as for the LSTM (see Fig. 4). The identification process was performed on CPU (Intel Core i7 CPU @ $2.80 \mathrm{GHz}$ with a RAM of $8 \mathrm{~GB}$ ) as there was no wide-spread library for a GPU-based training. The whole identification procedure of the DRDPI model took around 3.5 hours. A machine learning model i.e. Support 
Table II

HYPERPARAMETERS FOR THE LSTM NETWORK

\begin{tabular}{ccccccccc}
\hline \hline & $\begin{array}{c}\text { Number of } \\
\text { hidden layers }\end{array}$ & $\begin{array}{c}\text { Number of neurons } \\
\text { per cell }\end{array}$ & $\begin{array}{c}\text { Activation } \\
\text { functions }\end{array}$ & Optimizer & Loss function & $\begin{array}{c}\text { Training-subset } \\
\text { /Validation ratio }\end{array}$ & Batch size & Learning Rate \\
\hline LSTM & 2 & 64,64 & Tanh/Sigmoid & Adam & L2 Loss & $70 \% 30 \%$ & 16 & 0.001 \\
\hline
\end{tabular}
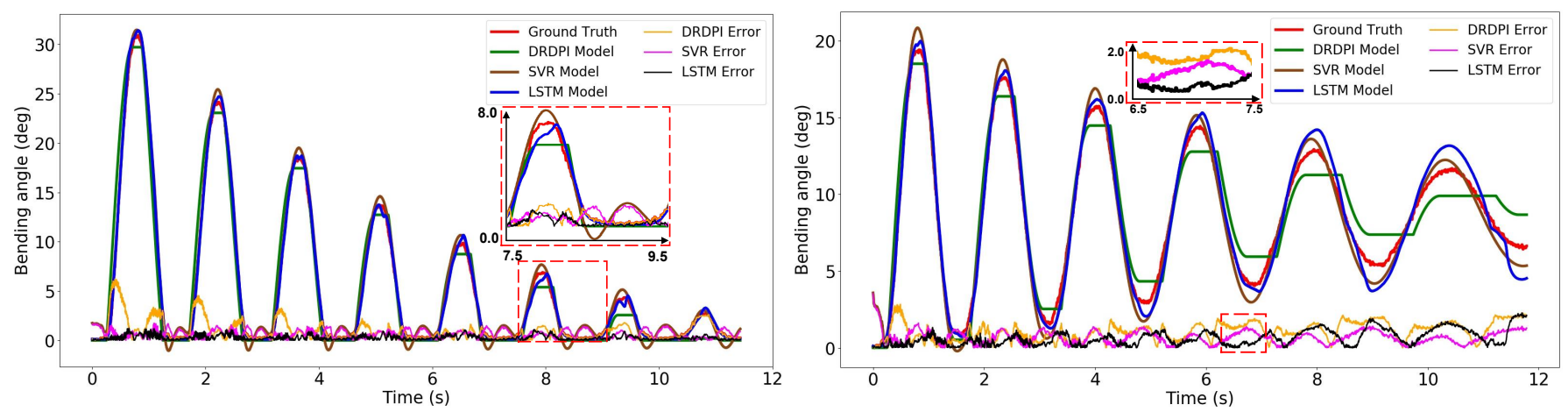

(a) Descending sine wave differing from training data $(f=0.7, \tau=0.12)$

(b) Attenuated down-chirp sine wave with shifted baseline $(\tau=0.1, c=-0.1)$
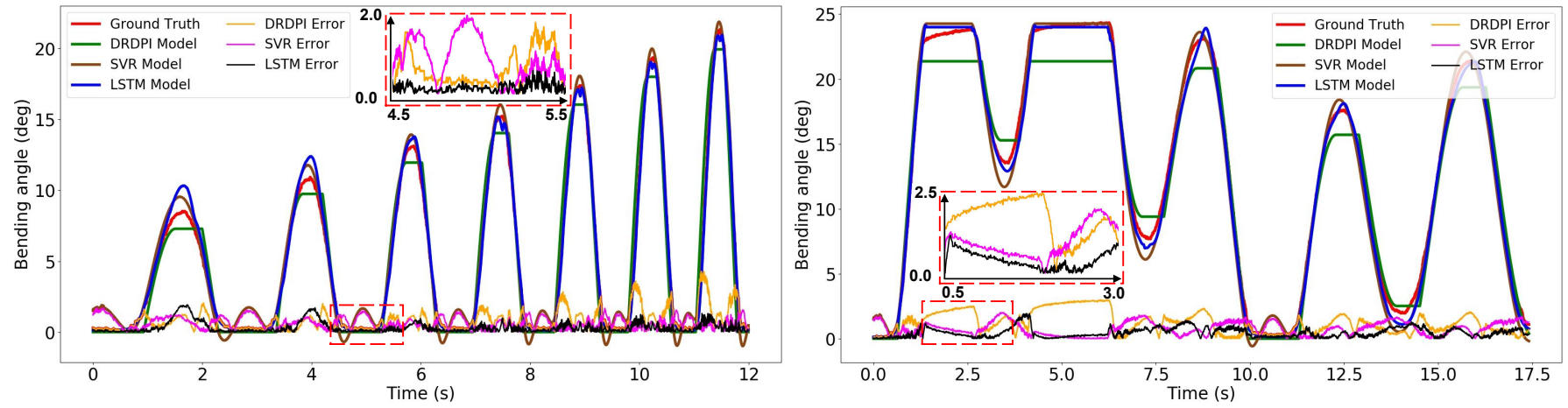

(c) Ascending up-chirp sine wave with zero baseline ( $f=0.3, \tau=0.05, c=0.15)$

(d) Arbitrarily varying signal with plateaus

Figure 5. The modeling performance of the LSTM (blue), SVR (brown) and DRDPI model (green) on four types of test signals. Modeling errors of the LSTM (black), SVR (magenta) and the DRDPI model (orange) are also visualised on the same plot for comparison.

Table III

QUANTITATIVE PERFORMANCE OF THE LSTM, THE SVR AND THE DRDPI MODEL ON FOUR GROUPS OF TEST SETS.

\begin{tabular}{|c|c|c|c|c|c|c|c|c|c|c|c|c|}
\hline \multirow{2}{*}{$\begin{array}{l}\text { Modeling } \\
\text { Methods }\end{array}$} & \multicolumn{3}{|c|}{$\begin{array}{c}\text { Descending sine wave } \\
\text { differing from training data }\end{array}$} & \multicolumn{3}{|c|}{$\begin{array}{c}\text { Attenuated down-chirp } \\
\text { sine wave with shifted baseline }\end{array}$} & \multicolumn{3}{|c|}{$\begin{array}{l}\text { Ascending up-chirp sine } \\
\text { wave with zero baseline }\end{array}$} & \multicolumn{3}{|c|}{$\begin{array}{l}\text { Arbitrarily varying } \\
\text { signal with plateaus }\end{array}$} \\
\hline & RMSE (deg) & MAE (deg) & NRMSE & RMSE (deg) & MAE (deg) & NRMSE & RMSE (deg) & MAE (deg) & NRMSE & RMSE (deg) & MAE (deg) & NRMSE \\
\hline DRDPI & 1.40 & 6.23 & $4.54 \%$ & 1.22 & 2.83 & $6.28 \%$ & 1.07 & 4.28 & $4.99 \%$ & 1.48 & 2.98 & $6.07 \%$ \\
\hline SVR & 0.76 & 1.83 & $2.45 \%$ & 0.80 & 3.54 & $4.10 \%$ & 0.76 & 1.69 & $3.56 \%$ & 0.92 & 2.01 & $3.78 \%$ \\
\hline LSTM & 0.36 & 1.23 & $1.17 \%$ & 0.82 & 2.27 & $4.20 \%$ & 0.50 & 1.92 & $2.34 \%$ & 0.59 & 1.93 & $2.42 \%$ \\
\hline
\end{tabular}

Vector Regression (SVR) was also implemented to enrich the comparison. The SVR is an expansion of a classical Support Vector Machine (SVM) from pure classification to regression tasks. Similar to SVM it is designed for estimation of high dimensional, nonlinear problems when only a limited number of samples are available [26]. The SVR model implemented in this paper was based on [26]. The hyperparameters of the SVR are chosen as follows: penalty $\mathrm{C}=10$, kernel $=$ Radial Basis Function (RBF) kernel, kernel coefficient $\gamma=0.1$, margin of tolerance $\epsilon=0.1$. Parameter $\mathrm{C}$ and $\gamma$ were optimized using a grid search $(\mathrm{C} \in\{0.001,0.01,0.1,1,10\}, \gamma \in\{0.001,0.01,0.1,1\})$ and a cross-validation method $(\mathrm{k}=10)$. The optimization results of the three models are displayed in Table IV. The rightmost column represents the mean of the relative change of the MSE loss in the last $10 \%$ of the iterations. Small mean of relative change indicates that the three models were adequately converged and the comparison is thus fair.

The pattern of the test signals as well as the modeling results of the LSTM, the DRDPI model, and the SVR are shown in Fig. 5 and described in detail as follows:
Table IV

OPTIMIZATION RESULTS OF THREE MODELS

\begin{tabular}{|c|c|c|c|}
\hline Modeling Methods & Iterations & Training Loss $\left(\mathrm{deg}^{2}\right)$ & Mean of Relative Change \\
\hline DRDPI & $\mathbf{1 0 0 0}$ & $\mathbf{0 . 3 4 4}$ & $\mathbf{0 . 0 1 3 \%}$ \\
\hline SVR & $\mathbf{1 0 0}$ & $\mathbf{0 . 0 0 2 7 8 2}$ & $\mathbf{0 . 7 0 8 \%}$ \\
\hline LSTM & $\mathbf{5 0}$ & $\mathbf{0 . 0 0 0 5 6}$ & $\mathbf{0 . 1 9 0 \%}$ \\
\hline
\end{tabular}

a): A descending sine wave following (5) with $f=0.7$ and $\tau=0.12$ differing from the training data was generated, while the amplitude $\mathrm{A}=1.5$ remains the same.

The RMSE and the NRMSE of the LSTM are $0.36^{\circ}$ and $1.17 \%$, respectively. Both metrics outperform those achieved by the DRDPI model $\left(1.40^{\circ}\right.$ and $\left.4.54 \%\right)$ and the SVR model. The error of the LSTM consistently remains under $1.23^{\circ}$ (MAE) across the whole range, which is even smaller than the RMSE of the DRDPI model.

$b)$ : To test the performance of the LSTM on data with time-varying frequency, a pressure signal following (10) with $A=0.9, B=1.2, f=0.7, \tau=0.1$ and $c=-0.1$ was utilized to generate attenuated down-chirp sine wave with nonzero baseline. The variable $c$ is chirpyness that regulates the changing rate of frequency over time.

$$
p(t)=A e^{-\tau t}\left(\sin \left(2 \pi(f+c t) t-\frac{\pi}{2}\right)+1\right)+B \quad[\text { bar }] \quad(10)
$$


Unlike the DRDPI model that can merely model the saturating area by using a plateau, the LSTM and the SVR is able to predict a smooth sine-shaped curve when approaching extrema. This phenomenon can also been observed from other test signals. Consequently, it leads to lower RMSEs of the LSTM and the SVR with $0.82^{\circ}$ and $0.80^{\circ}$, respectively compared to the DRDPI with $1.22^{\circ}$.

c): The third set of test data has an ascending pattern that is not contained in the training data. Following (11), with $A$ $=0.6, f=0.3, \tau=0.05$ and $c=0.15$, an ascending up-chirp sine wave was generated.

$$
p(t)=A e^{-\tau t}\left(\sin \left(2 \pi(f+c t) t-\frac{\pi}{2}\right)+1\right) \quad[\mathrm{bar}]
$$

The performance of the LSTM advances with growing frequency, whereas the DRDPI model achieves a similar level of performance in the first two loops, before it starts to deteriorate until reaching an MAE of $4.28^{\circ}$ in the loading phase of the last loop. Note that the SVR consistenly predicts wavy lines in deadzones, causing its performance to be $34.2 \%$ worse than the LSTM in terms of the RMSE.

d): Arbitrarily varying signals represent the most general commands that can take place in practice. It is used to further explore the potential of the LSTM.

The LSTM shows consistently low prediction errors, regardless of the signal patterns, with an RMSE and NRMSE of $0.59^{\circ}$ and $2.42 \%$, respectively. While the DRDPI also predicts a quasi-plateau, it can be seen that each plateau happens at a considerable offset (Fig. 5d), where its MAE of $2.98^{\circ}$ emerges on the second plateau. Both the RMSE $\left(0.59^{\circ}\right)$ and the NRMSE $(2.42 \%)$ of the LSTM are $60.1 \%$ and $36.0 \%$ smaller than the DRDPI model and the SVR, respectively.

The average inference time of the LSTM for a single point is approximately $25 \mathrm{~ms}$ based on the test data. Table III summarizes and compares the performance of the three models quantitatively based upon the three metrics. In terms of NRMSE, apart from group (b) in which the LSTM is $33.1 \%$ better than the DRDPI model, the LSTM outperforms the DRDPI model by at least $50 \%$ in the other three groups. Moveover, except for achieving a comparable performance in the ascending up-chirp sine wave, the LSTM is at least $34.2 \%$ better than the SVR. With the provided experiments, we showcased that the LSTM is capable to capture dynamic responses of our system which are - given that our catheter is lightweight and the speeds are moderate - dominated by the valve dynamics. We believe that faster motion could be captured also if trained properly. Overall, the LSTM is able to accurately model the asymmetric, saturated and rate-dependent hysteresis originating from the entire catheter system with a sub-degree precision as well as a lead in performance compared to the DRDPI model and the SVR. In cardiovascular applications, the required precision that clinicians indicate as being acceptable is typically in the order of 1-3 mm [27], which corresponds to 2.09-6.26 degrees according to (4). Although confirmed in a simplified setting, the obtained performance of the LSTM (see Table III) shows a good potential to satisfy the requirements in terms of precision also in a more realistic clinical setting.

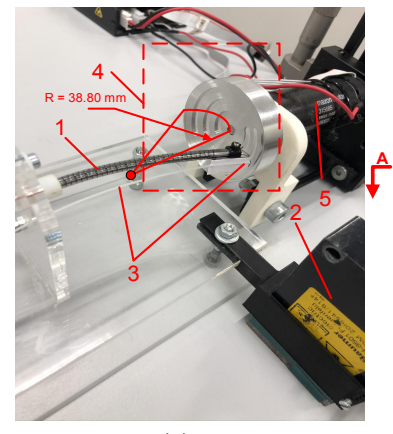

(a)

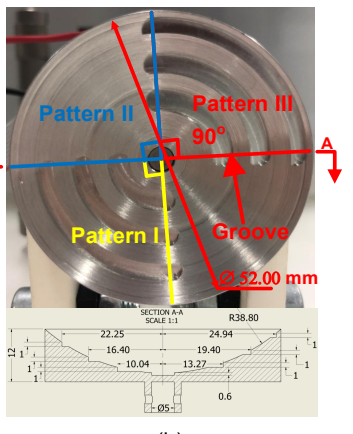

(b)
Figure 6. (a) overview of reconstruction module: 1. distal catheter segment; 2. pneumatic artificial muscle; 3. OCT fiber; 4. reconstruction Object; 5. DC motor; (b) (up) three patterns distributed in a circular sector of $270^{\circ}$ (bottom) cross-section view.

\section{B. 3D Reconstruction Experiment}

In a bifurcation lesion, angiography may not be able to accurately image the carina area because the main vessel and side branch overlap with each other. As a solution, a catheter-based forward-looking intravascular ultrasound (FLIVUS) could help reconstruct the anatomy of the lesion [28]. In this case, the positioning accuracy of the catheter tip is very critical otherwise a precise $3 \mathrm{D}$ reconstruction can not be guaranteed. A 3D reconstruction experiment is depicted in this subsection (Fig. 6). A forward-looking optical coherence tomography (FL-OCT) fiber is used to simulate the A-mode FL-IVUS. The experiment is set up to test whether the modeling accuracy of the LSTM allows high quality reconstruction of the environment. Considering that LSTM and SVR both rely on machine-learning, while DRDPI is analytic and given superiority of LSTM over SVR it was found more interesting to compare the performance of the LSTM with the analytic DRDPI in the following experiment.

The experimental setup is shown in Fig. 2. Apart from the modules introduced in section II-B, a reconstruction module is added for this experiment. A hollow spherical cap with embedded grooves (Fig. 6) is fabricated to serve as a dummy environment. This cap is positioned in front of the catheter tip. There are three patterns on this object, and each of them covers a circular sector of $90^{\circ}$. The dimensions of the object as well as the patterns can be seen in Fig. 6. The pattern I and III have three milled grooves at various distances with respect to the center axis, while pattern II is a smooth surface without any grooves. The object is rotated by a DC motor (Maxon Group, Switzerland) so as to simulate the rotation motion of the catheter. An OCT fiber (iOCT, OptoMedical Technologies $\mathrm{GmbH}$, Germany) is attached onto the catheter tip to measure the front distance to the object by using an A-mode scan. The acquired raw A-mode images are published on a ROS topic and processed in Matlab (Fig. 2) using a method introduced in [29]. A harmonic sinusoidal pressure $(A=2.95$ [bar], $f=0.1$ $\mathrm{Hz}$ ) is used to bend the catheter in order to cover the whole range of the semi spherical cap. The scan object remains still during one back and forth motion of the catheter, and then rotates for $15^{\circ}$ for the next scan until all the three patterns are scanned. The lateral distance sensor measures the actual tip bending angle. By combining the bending angle measured by the laser with the OCT measurement, followed by a conversion 
(a)
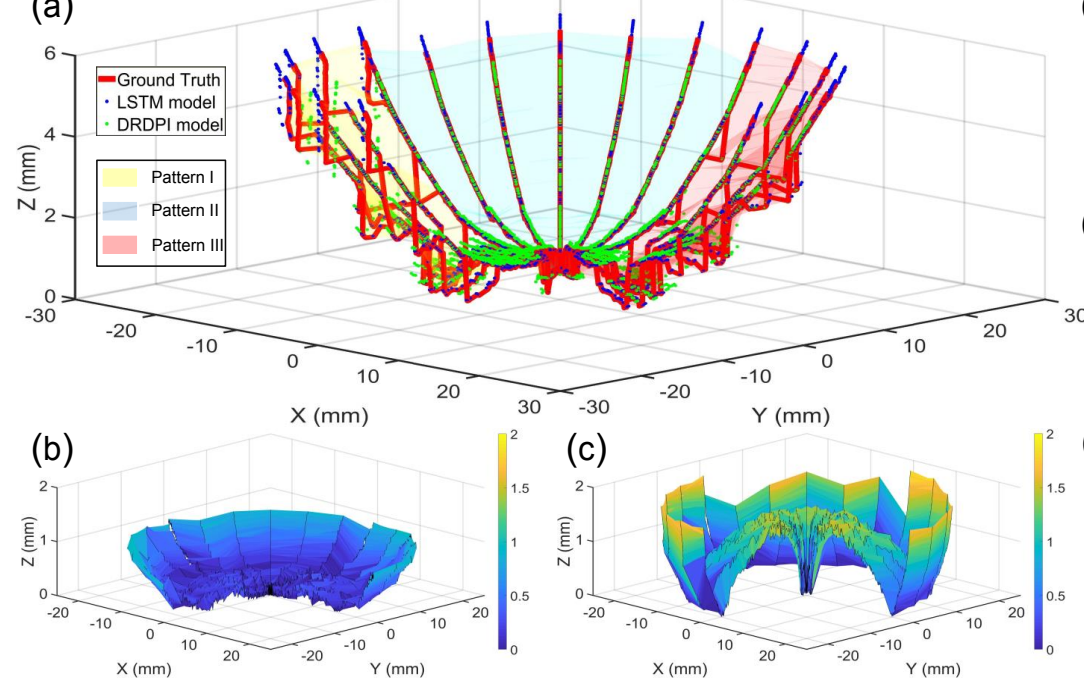

(c)

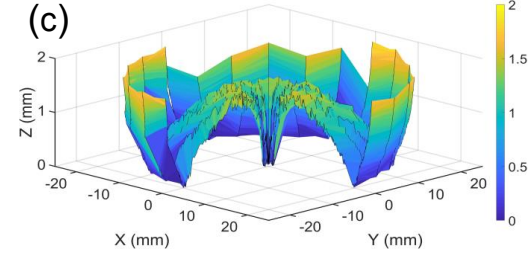

(d)

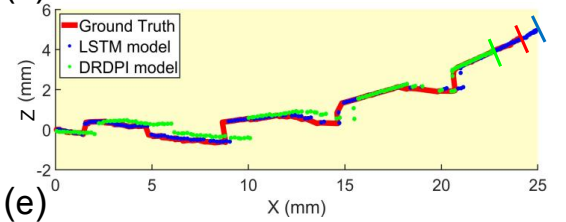

(o)

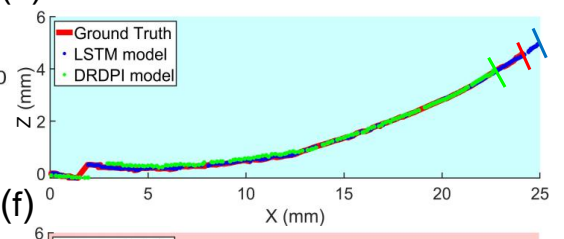

(

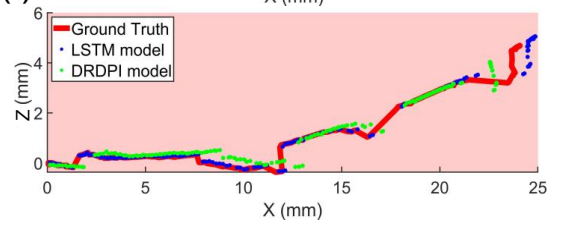

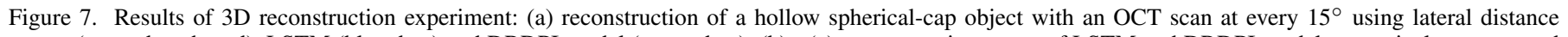

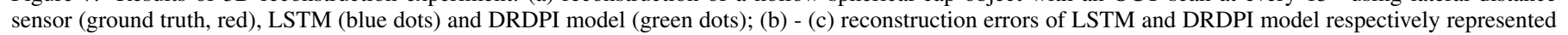
in color-coded maps; (d) - (f) middle cross-section (at $45^{\circ}$ ) of each pattern showing the planar reconstruction performance of the two models. Table V

\begin{tabular}{|c|c|c|c|c|c|c|c|c|c|c|c|c|c|c|c|}
\hline \multirow{2}{*}{$\begin{array}{c}\text { Angle } \\
\left({ }^{\circ}\right)\end{array}$} & \multirow{11}{*}{$\begin{array}{l}\text { Pattern } \\
\text { I }\end{array}$} & \multicolumn{2}{|c|}{ LSTM } & \multicolumn{2}{|c|}{ DRDPI } & & \multicolumn{2}{|c|}{ LSTM } & \multicolumn{2}{|c|}{ DRDPI } & & \multicolumn{2}{|c|}{ LSTM } & \multicolumn{2}{|c|}{ DRDPI } \\
\hline & & Mean & Std & Mean & Std & & Mean & Std & Mean & Std & & Mean & Std & Mean & Std \\
\hline 0 & & 0.15 & 0.16 & 0.54 & 0.46 & & 0.14 & 0.16 & 0.53 & 0.42 & & 0.24 & 0.29 & 0.69 & 0.57 \\
\hline 15 & & 0.18 & 0.21 & 0.57 & 0.46 & & 0.14 & 0.17 & 0.52 & 0.41 & & 0.26 & 0.30 & 0.73 & 0.59 \\
\hline 30 & & 0.11 & 0.13 & 0.50 & 0.41 & Dotton & 0.19 & 0.21 & 0.54 & 0.45 & & 0.24 & 0.29 & 0.73 & 0.58 \\
\hline 45 & & 0.22 & 0.25 & 0.58 & 0.47 & Patern & 0.15 & 0.19 & 0.53 & 0.43 & Patlern & 0.26 & 0.32 & 0.67 & 0.56 \\
\hline 60 & & 0.18 & 0.21 & 0.51 & 0.44 & & 0.18 & 0.21 & 0.56 & 0.43 & & 0.27 & 0.31 & 0.68 & 0.57 \\
\hline 75 & & 0.15 & 0.21 & 0.56 & 0.42 & & 0.14 & 0.17 & 0.54 & 0.41 & & 0.22 & 0.28 & 0.64 & 0.54 \\
\hline 90 & & 0.14 & 0.18 & 0.52 & 0.42 & & 0.17 & 0.19 & 0.55 & 0.45 & & 0.24 & 0.29 & 0.69 & 0.57 \\
\hline Overall & & 0.16 & 0.04 & 0.54 & 0.03 & & 0.16 & 0.02 & 0.54 & 0.01 & & 0.25 & 0.02 & 0.69 & 0.03 \\
\hline p-value & & & 0.0 & 117 & & & & & 2021 & & & & 0.0 & 998 & \\
\hline
\end{tabular}

from Polar to Cartesian Coordinate, an approximation of the ground truth profile of the environment is obtained. This profile is then compared with the environment that is computed from the modeled bending angle (LSTM and DRDPI respectively) and the OCT distance measurement. If the hysteresis were compensated perfectly, both reconstructions should match. The reconstruction error is defined as the mean of the Euclidean distance between the ground truth and the reconstructed points derived by the two models.

The overall reconstruction performance is shown in Fig. 7a. A quantitative evaluation can be found in Table V. Figure $7 \mathrm{~d}$ shows the 45 degree planar reconstruction of pattern I. One can observe that the LSTM can reconstruct the crosssection profile with high accuracy (reconstruction error = $0.22 \mathrm{~mm}$, Table V). Both the shape and the location of the grooves are well-reconstructed except that the reconstructed curve is slightly stretched at the end with respect to the ground truth. In contrast, the DRDPI model achieves an acceptable performance on curve, whereas the locations of the first and second grooves are reconstructed with a large variation, leading to a large reconstruction error of $0.58 \mathrm{~mm}$ (Table V). In Fig. 7e and Fig. 7f, the reconstruction performance of the two models follow a similar trend in Pattern I. The color-coded maps in Fig.7b and Fig. 7c describe the reconstruction error across all the patterns. The reconstruction error of the LSTM gradually rises up until reaching around $1 \mathrm{~mm}$ when approaching the edge of the object. Unlike the LSTM, the DRDPI model has a large reconstruction error around the center axis as it utilizes deadband operators to model the dead zone of the hysteresis. After achieving its best performance in the halfway, its performance degrades again and the reconstruction error approaches ca. $2 \mathrm{~mm}$. The reason for that is explained as follows. Unlike the LSTM can track the area near upper turning point with a smooth sine-shaped curve, the DRDPI model still use deadband operators i.e. plateaus to model the upper turning point which lead a large modeling error. As shown in Table III, the average reconstruction error of the LSTM are $0.16 \mathrm{~mm}$, $0.16 \mathrm{~mm}$ and $0.25 \mathrm{~mm}$ in pattern I to III. These errors are $70.4 \%, 70.4 \%, 63.8 \%$ lower than those of the DRDPI model. To make sure that the difference in results between the two models are not caused by sampling errors, a Mann-Whitney U Test is used to compare the LSTM and the DRDPI model. The null hypothesis $H_{0}$ that the mean reconstruction errors of the two models were not statistically different, could be rejected as a p-value smaller than 0.01 was found in all the three patterns. The results prove that performance of the LSTM and the DRDPI model are statistically significantly different.

The reconstruction experiment validate the feasibility that, with the assistance of the LSTM, a PAM-driven catheter is able to overcome the complex inherent hysteresis and accurately reconstruct the anatomical structure with a forward-looking imaging modalities in intravascular interventions. 


\section{CONCLUSIONS AND FUTURE WORK}

To address hysteresis problem in robotic catheters, a LSTM network is proposed to model the hysteresis. To this end, an experimental setup containing a one-DOF PAM-driven catheter segment was developed for model validation. Descending sinusoidal pressure signals under four frequencies were used to generate multi-loop hysteresis, which was further used as training data for the LSTM. The acquired data reveal a complex pattern namely asymmetric, saturated, rate-dependent hysteresis, which originate from the entire catheter system rather than the PAM itself. A DRDPI model and a SVR model were established for comparison to the LSTM.

The LSTM was first validated on four test signals containing diverse patterns e.g. chirp signals, arbitrarily varying signals. The RMSE and MAE of the LSTM remain under $0.82^{\circ}, 2.27^{\circ}$, respectively across different test signals. The results reveal that the LSTM performs better than the DRDPI model and the SVR. Next, the LSTM was further validated through an OCT reconstruction experiment. The results indicate that the LSTM, with its good modeling accuracy, is able to reconstruct the object with errors below $0.25 \mathrm{~mm}$ in all of the three patterns, while the smallest reconstruction error achieved by the DRDPI model is $0.54 \mathrm{~mm}$.

In general, with the LSTM, the response of the catheter tip (bending angle) can be predicted with sub-degree precision solely based on the input commands (the pressure at proximal end). It demonstrates its robustness when the catheter system exhibits a complex hysteresis. The proposed method requires similar amount of training data compared to analytical models and machine learning methods, and is very fast to characterize. The LSTM reduces the need to mount a sensor at the distal tip for localization, which is originally challenging due to spatial limitation and sterilization requirement. The proposed LSTM makes it possible to steer robotic catheters with good precision. We demonstrated this ability in a free space scenario (representing motion in the heart), which could enable interesting clinical applications such as detailed intra-operative reconstruction of anatomic lumens or cavities. Although validated in a relatively simple 1-DOF planar bending system, our model could be readily extended to 2-DOF spatial bending with four antagonistic PAMs and four LSTMs identified by the four PAMs. A single DOF bending in combination with rotational motion about the catheter's longitudinal axis could in fact suffice as well, but in reality the rotational motion would also need to be trained.

Future work will focus on embedding an LSTM network in controllers that actually compensate for the hysteresis on the PAM-driven catheters. Moreover, the proposed LSTM is a generic method and its generalization ability on other systems suffering from hysteresis will also be investigated.

\section{REFERENCES}

[1] E. Kandaswamy and L. Zuo, "Recent advances in treatment of coronary artery disease: role of science and technology," Int. J. of Mol. Sci., Vol. 19, No.2, pp.424.

[2] P. S. Douglas, et al., "Outcomes of Anatomical versus Functional Testing for Coronary Artery Disease", The new england journal of medicine, Vol. 372, No. 14, pp. 3-18., 2015

[3] K. Yoshimitsu et al., "A novel four-wire-driven robotic catheter for radiofrequency ablation treatment", Int. J. of Comput. assisted Radiol. and surgery, Vol. 9, No. 5, pp.867-874, Feb. 2014
[4] Y. Chen et al., "Modal-based kinematics and contact detection of soft robots", Soft robotics, July, 2020, doi:10.1089/soro.2019.0095.

[5] I.S. Godage, et al. "Dynamic Control of Pneumatic Muscle Actuators", IEEE IROS Workshop:Soft Robotic Modeling and Control, Oct. 2018.

[6] M. De Volder, A. J.M. Moers, and D. Reynaerts, "Fabrication and control of miniature McKibben actuators," Sensors and Actuators, A:Physical, vol. 166, no. 1, pp. 111-116, Mar. 2011.

[7] B. Bardou et al., "Improvements in the control of a flexible endoscopic system". In IEEE Int. Conf. on Robot. and Automat. (ICRA), 2012.

[8] R.A. Porto et al., "Position control of medical cable-driven flexible instruments by combining machine learning and kinematic analysis", In Int. Conf. on Robot. and Automat. (ICRA), pp. 7913-7919, 2019

[9] P. Cabras et al., "An adaptive and fully automatic method for estimating the 3D position of bendable instruments using endoscopic images", The Int. J. of Med. Robot. and Comput. Assisted Surgery, p.e1812, 2017

[10] R. Reilink et al., "3D position estimation of flexible instruments: markerless and marker-based methods", International journal of computer assisted radiology and surgery, Vol. 8, No.3, pp.407-417,2013

[11] D. Baek et al., "Hysteresis Compensator with Learning-Based Hybrid Joint Angle Estimation for Flexible Surgery Robots", IEEE Robotics and Automation Letters, Vol. 5, No. 4, Oct., 2020

[12] O. M. Omisore et al., "Learning-based Parameter Estimation for Hysteresis Modeling in Robotic Catheterization”, 41st Annu. Int. Conf. of the IEEE Eng. in Medicine and Biol. Soc., pp. 5399-5402, 2019

[13] K. Choi et al., "A hybrid dynamic model for the AMBIDEX tendondriven manipulator". Mechatronics, 69, p.102398, 2020

[14] X. Li et al., "Distal-end force prediction of tendon-sheath mechanisms for flexible endoscopic surgical robots using deep learning". Mechanism and Machine Theory, 134, pp.323-337, 2019

[15] X. Li, et al., "Deep learning for haptic feedback of flexible endoscopic robot without prior knowledge on sheath configuration". Int. J. of Mech. Sciences, 163, p.105129, 2019

[16] N. Hirose et al., "Modeling of rolling friction by recurrent neural network using LSTM". IEEE International Conference on Robotics and Automation (ICRA), pp. 6471-6478, 2017

[17] T.N. Do et al., "Hysteresis modeling and position control of tendonsheath mechanism in flexible endoscopic systems", Mechatronics, Vol. 24, No.1 , pp.12-22, 2014

[18] O. M. Omisore et al., "Towards Characterization and Adaptive Compensation of Backlash in a Novel Robotic Catheter System for Cardiovascular Interventions," in IEEE Transactions on Biomedical Circuits and Systems, vol. 12, no. 4, pp. 824-838, Aug. 2018.

[19] J. Legrand, et al. "Active Handheld Flexible Fetoscope-Design and Control Based on a Modified Generalized Prandtl-Ishlinski Model",In 2020 IEEE/ASME Int. Conf. on Advanced Intell. Mechatronics (AIM), pp. 367-374, Jul., 2020

[20] W. Xu , et al. "Data-driven methods towards learning the highly nonlinear inverse kinematics of tendon-driven surgical manipulator," The Int. J. of Med. Robot. and Comput. Assisted Surgery, Vol.13, 2017

[21] R.J. Webster III, and B.A. Jones, "Design and kinematic modeling of constant curvature continuum robots: A review", The International Journal of Robotics Research, Vol. 29, No.13, pp.1661-1683, 2010

[22] V. Hassani et al., "A survey on hysteresis modeling, identification and control," Mech. Syst. and Signal Process., Vol. 49, pp.209-233, 2014.

[23] S. Hochreiter and J. Schmidhuber, "Long Short-Term Memory," Neural Computation, vol. 9, no. 8, pp. 1735-1780, Nov. 1997.

[24] D. Wu et al., "Towards Modeling of Hysteresis in Robotic Catheters based on LSTM", 32nd Annual Conference of the International Society for Medical Innovation and Technology (iSMIT), 2020.

[25] S. Shakibaet al., "Modeling and compensation of asymmetric ratedependent hysteresis of a miniature pneumatic artificial muscle-based catheter". Mechanical Systems and Signal Processing, Vol. 154, 2021.

[26] S. Zhang et al., "An Easy-to-Implement Hysteresis Model Identification Method Based on Support Vector Regression", IEEE Transactions on Magnetics, Vol. 53, No. 11, pp. 1-4, 2017.

[27] F. Bourier et al., "Accuracy assessment of catheter guidance technology in electrophysiology procedures: A comparison of a new 3D-based fluoroscopy navigation system to current electroanatomic mapping systems", J. Cardiovascular Electrophysiology, pp. 74-83, 2014.

[28] J. Janjic et al., "Sparse Ultrasound Image Reconstruction From a ShapeSensing Single-Element Forward-Looking Catheter", IEEE Transactions on Biomedical Engineering, Vol. 65, No. 10, 2018

[29] J. Smits et al., "Development and Experimental Validation of a Combined FBG Forceand OCT Distance Sensing Needle for Robot-Assisted Retinal Vein Cannulation", IEEE International Conference on Robotics and Automation (ICRA), May, 2018. 